\title{
MECHANICAL ANNEALING MODEL OF DAMAGE IN ARRAYS OF NANOPILLARS
}

\author{
Tomasz Derda \\ Institute of Mathematics, Czestochowa University of Technology \\ Czestochowa, Poland \\ tomasz.derda@im.pcz.pl
}

\begin{abstract}
The mechanical damage accumulation occurring in an array of axially loaded nanopillars is studied within the stochastic approach. To each nanopillar, an initial strength-threshold is assigned which is drawn from the Weibull probability distribution. Under the influence of load exceeding the strength-threshold the pillar height is reduced. Then the reduced pillar gets new higher strength-threshold and its load is transferred to other working pillars. We analyse slip loads causing the system height reductions and avalanches of the pillar height reductions.
\end{abstract}

Keywords: nanopillars, fibre bundle model, mechanical annealing

\section{Introduction}

In recent years many tensile and compressive experiments have been performed on nano- and microscale metallic pillars [1-3]. The materials under investigation are for instance nickel, gold, copper, molybdenum and aluminium. These experiments on nano-sized pillars confirm substantial strength-increase via the size reduction of the sample [3]. In works [4,5] concerning compression tests of nanosized nickel and molybdenum pillars a process called "mechanical annealing" has been observed. Scientists dealing with these compression experiments noticed that before the tests the nanopillars were full of dislocations. During the "mechanical annealing" process density of dislocations in the pillar was reduced by many orders of magnitude producing an almost dislocation-free crystal. Therefore pillars exhibited enhanced strength and progressively higher stresses are required in order to continue deformation.

Studies on arrays of free-standing nanopillars subjected to uniaxial microcompression reveal the potential applicability of nanopillars as components for the fabrication of micro- and nano-electromechanical systems, micro-actuators or optoelectronic devices $[3,6]$. This work concerns a partial damage process in a set of pillars assembled to a flat and rigid substrate. Such an arrangement is applied in systems of micromechanical sensors. The model described below is based on the idea of a fibre bundle model with stick-slip dynamics [7, 8]. 


\section{Description of the model}

The system under consideration consists of $N$ parallel nanopillars. The terms "nanopillar" and "pillar" are used interchangeably. The initial height of each nanopillar equals $h_{i}(\tau=0)=1, i=1,2, \ldots, N$, therefore the initial height of the system takes value $H(\tau=0)=1$. Here, $\tau$ denotes the number of time step. To each nanopillar $x_{i}$ we assign an initial critical load $\sigma_{t h}^{i}(\tau=0), i=1,2, \ldots, N$, which is a strength-threshold of the given nanopillar subjected to axial loading. Initial strength-thresholds $\sigma_{t h}^{i}(\tau=0), i=1,2, \ldots, N$ are independent and identically distributed random variables with the probability density and distribution functions: $p\left(\sigma_{t h}\right), P\left(\sigma_{t h}\right)$. It is assumed that the randomness of initial strength-thresholds represents the disorder of heterogeneous material. The variety of pillar strength-thresholds results from fabrication errors of individual pillars. In this paper we employ the Weibull distribution:

$$
P\left(\sigma_{t h}\right)=1-\exp \left[-\left(\frac{\sigma_{t h}}{\lambda}\right)^{\rho}\right]
$$

Here, the dimensionless parameter $\rho$ is the Weibull index and $\lambda$ is a scale parameter (setting the scale of the thresholds), which is fixed to $\lambda=1$ in this work. The Weibull index controls the amount of disorder in the initial pillar strength-thresholds, so it is the model's key parameter. The bigger the Weibull index $\rho$, the smaller the disorder.

The Weibull distribution is widely used in materials science, because conducted experiments proved that real materials follow the Weibull probability distribution functions very closely for strength and time to damage of individual elements [9]. It is assumed in materials science that $\rho \in\langle 2,10\rangle[10]$.

The system is subjected to an axial external load $F$ increased in a quasi-static way. All the nanopillars are intact at the beginning of the loading process, so $F$ grows gradually from $F(\tau=0)=0$ and the load of nanopillars changes with time in accordance with relation $\sigma(\tau)=F(\tau) / N_{l}(\tau)$, where $N_{l}(\tau)$ is the number of currently loaded (working) nanopillars.

When load $\sigma_{i}>\sigma_{t h}^{i}$ the pillar height is irreversibly and instantly reduced

$$
h_{i}(\tau)=h_{i}(\tau-1)-k \cdot \Delta h
$$

Coefficient $k$ takes value:

$$
k=\left\{\begin{array}{ccc}
0 & \text { for } & \sigma_{i}(\tau) \leq \sigma_{t h}^{i}(\tau) \\
1 \text { or } 2 & \text { for } & \sigma_{i}(\tau)>\sigma_{t h}^{i}(\tau)
\end{array}\right.
$$


The parameter $\Delta h$ is associated with degree of the pillar height reduction and its value, in this work, is $\Delta h=0.01$. Therefore, single pillar height reduction takes a random value 0.01 or 0.02 . The cumulative value of pillar height reductions at time step $\tau$ is equal to $h_{i}^{\text {red }}(\tau)=h_{i}(0)-h_{i}(\tau)$.

As a result of the pillar height reduction, because of elimination of defects from the crystal, the reduced pillar gets a higher strength-threshold:

$$
\begin{gathered}
\sigma_{t h}^{i}(\tau)=\sigma_{t h}^{i}(\tau-1) \cdot(\delta+1) \\
\delta=\eta \cdot k \cdot \Delta h \cdot 100
\end{gathered}
$$

here, the factor $\eta$ is a strengthening coefficient of the reduced pillar. The coefficient $\eta$ is randomly drawn from the interval $\left[\eta_{\min }, \eta_{\max }\right]$ for each pillar reduction.

Let $h_{\max }(\tau)=\operatorname{Max}\left[h_{i}(\tau)\right]$ denote the height of the highest pillar at the time step $\tau$, hence the system height in this moment is $H(\tau)=h_{\max }(\tau)$ and reduction of initial system height takes value $H_{\text {red }}(\tau)=H(0)-h_{\max }(\tau)$. If the height of the reduced $i^{\text {th }}$ pillar $h_{i}(\tau) \leq H(\tau)-0.02$, then the pillar is not working and its load $\sigma_{i}$ has to be transferred to other working pillars. For the load transfer we assume an infinite range of interaction, which is represented by the global load sharing (GLS) rule. For this rule the increased loads caused by the pillar reduction are shared equally by all currently loaded pillars, so the load of the working pillars increases by $\sigma_{t r}=\sigma_{i} / N_{l}(\tau)$ and the load of the reduced non-working pillar falls to zero $\sigma_{i}=0$. All the working pillars are subjected to an equal load. If the height of the $i^{\text {th }}$ pillar after reduction is $h_{i}(\tau) \geq H(\tau)-0.01$ there is no load transfer, the pillar is still working and a stable state occurs.

A load transfer from reduced and non-working pillar may give rise to reductions of other working pillars due to loads exceeding the strength-thresholds of these pillars. Consequently, a subsequent load transfer from reduced pillars may cause further reductions. If the load transfer does not provoke the next pillar height reductions or heights of the reduced pillars $h_{i}(\tau) \geq H(\tau)-0.01$ there is a stable state.

To continue the damage process in the case of the stable state occurrence the external load $F$ has to be increased with a small amount $\delta \sigma$ for each working pillar. The load increase $\delta \sigma$ takes a minimum value to provoke height reduction of the weakest working pillar. The above-described procedure has to be repeated until first stable state occurs for $H(\tau) \leq 0.9$. This means that external load $F$ is increased only if the system height $H(\tau)>0.9$.

After the height reduction, each pillar obtains a new higher strength-threshold in accordance with formula (4). All the pillars whose heights $h_{i}(\tau) \geq H(\tau)-0.01$ are subjected to equal load $\sigma$. When the system height is decreased $H(\tau)<H(\tau-1)$, all the non-working pillars whose heights $h_{i}(\tau) \geq H(\tau)-0.01$ are again loaded by 
$\sigma=F / N_{l}(\tau)$. In this context, a so-called slip load is an external load $F$ that induces the height reduction $H(\tau)=H(\tau-1)-0.01$ of the whole system.

\section{Analysis of the chosen simulation results}

Simulations have been performed for several system sizes with strengthening coefficients uniformly drawn from the intervals: [0.05, 0.15], [0.1, 0.2], [0.15, 0.25], $[0.2,0.3]$ and $[0.25,0.35]$. We considered sets of pillars with initial random strength-thresholds $\sigma_{t h}^{i}(0), i=1,2, \ldots, N$ governed by the Weibull probability distribution. Damage of systems with different degrees of structural disorder (characterised by the Weibull index) has been examined, namely $\rho=2,5,7,10$.

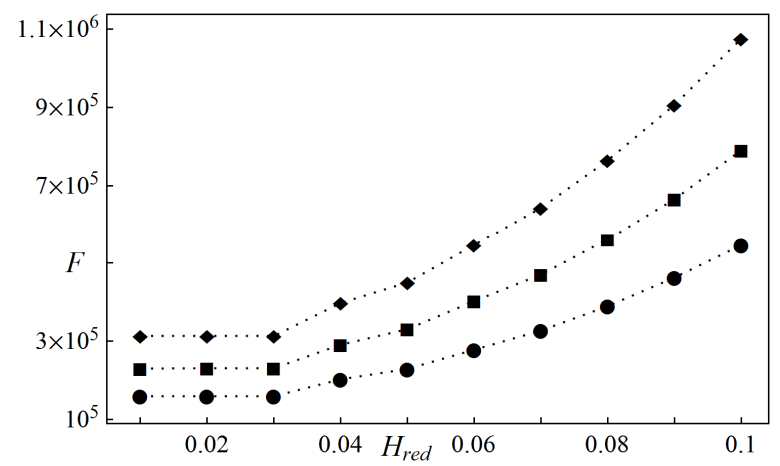

Fig. 1. Slip loads $F$ received for the subsequent system height reductions $H_{\text {red }}$. Results for single samples of sizes: $N=500 \times 500$ (circles), $N=600 \times 600$ (squares) and $N=700 \times 700$ (diamonds) pillars. Strengthening coefficients drawn from the interval $[0.15,0.25]$, Weibull index $\rho=5$

Figure 1 illustrates subsequent slip loads $F$ for single samples of sizes $N=500 \times 500, N=600 \times 600$ and $N=700 \times 700$ pillars. Strengthening coefficients are drawn from the interval $[0.15,0.25]$ and disorder is determined by Weibull index $\rho=5$. It can be seen that for all system sizes the first three height reductions are under equal or almost equal external load, despite a gradual increase of pillar strengths. The fourth consecutive system height reduction is induced by a considerably increased load. Further height reductions also needed an increase of external load $F$. After the rescaling of slip loads by adequate system size we received dimensionless values of slip loads $\sigma_{u}=F / N$. For samples showed in Figure 1 values of $\sigma_{u}$ are independent of the system size. Subsequent system height reductions are induced by nearly equal values of $\sigma_{u}$ irrespective of the system size.

Mean values of the subsequent slip loads $F$ for systems with different intervals $\left[\eta_{\min }, \eta_{\max }\right]$ are shown in Figure 2. The analysed systems consist of $N=50^{2}$ pillars and disorder of initial strength-thresholds is specified by the Weibull index $\rho=2$. 
Reductions of the system height $H_{\text {red }}>0.1$ (visible in Fig. 2) have to be explained as follows. When the height of the system $H \leq 0.9$, the external load $F$ is not increased. However, the current force $F$ is still applied and the loading process continues until the occurrence of the stable state. Hence the final system height may take values $H_{\text {final }}<0.9$.

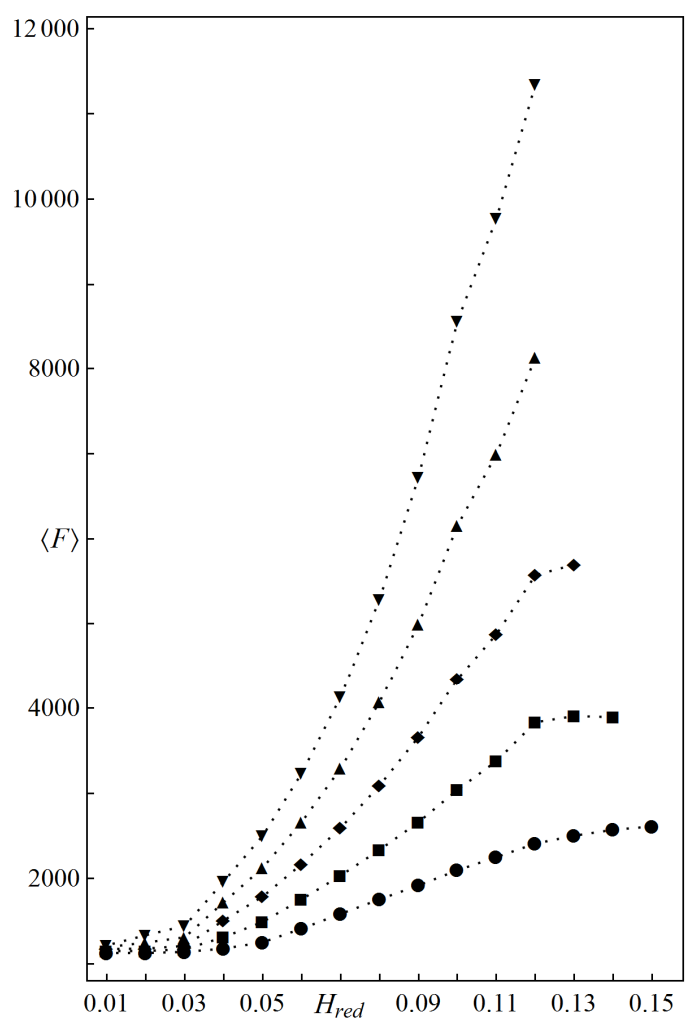

Fig. 2. Mean values of slip loads $F$ received for the consecutive system height reductions $H_{\text {red }}$. Comparison of the results obtained for different intervals $\left[\eta_{\min }, \eta_{\max }\right]:[0.05,0.15]$ (circles), [0.1, 0.2] (squares), [0.15, 0.25] (diamonds), $[0.2,0.3]$ (up triangles), $[0.25,0.35]$ (down triangles). System size $N=50 \times 50$ pillars, Weibull index $\rho=2$

With the increase of pillar strengths the mean values of consecutive slip loads for different intervals $\left[\eta_{\min }, \eta_{\max }\right]$ increasingly differ from each other. It can also be noticed that the bigger the strengthening coefficients $\eta$ are the more dynamic the increase of slip loads. From Figure 2 it can also be observed that the bigger the strengthening coefficients, the faster the systems attain stable states. For systems with coefficients $\eta$ drawn from the interval $[0.05,0.15]$ the height reductions are up to $H_{\text {red }}=0.15$. By contrast, for systems characterised by the interval $[0.25,0.35]$ maximum reductions do not exceed $H_{\text {red }}=0.11$. 
Analysing the results presented in Figure 3 the influence of strength-threshold disorder has to be noticed. The mean values of slip loads $F$ are ordered in accordance with the Weibull index $\rho$. The subsequent height reductions for a weakly disordered system $(\rho=10)$ are under the slip loads greater than for strongly disordered system $(\rho=2)$. A characteristic of increase of slip loads is similar for all investigated disorder degrees.

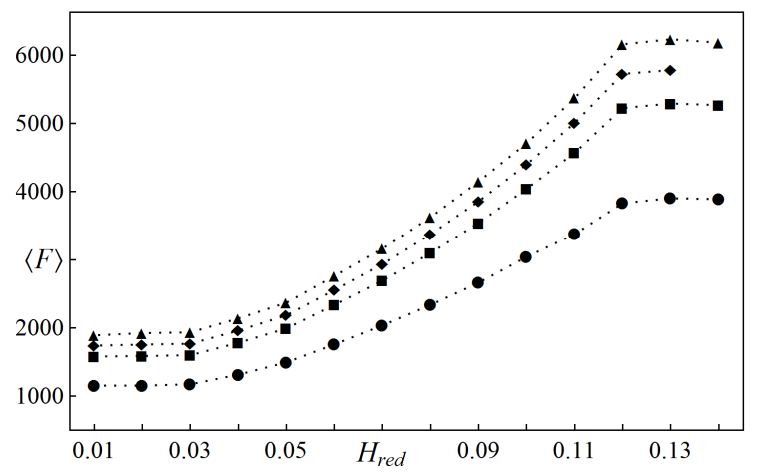

Fig. 3. The mean values of slip loads $F$ for subsequent height reductions $H_{\text {red }}$. Comparison of pillar strength-threshold disorder: $\rho=2$ (circles), $\rho=5$ (squares), $\rho=7$ (diamonds), $\rho=10$ (triangles). System size $N=50 \times 50$ pillars, coefficients $\eta$ drawn from the interval $[0.1,0.2]$

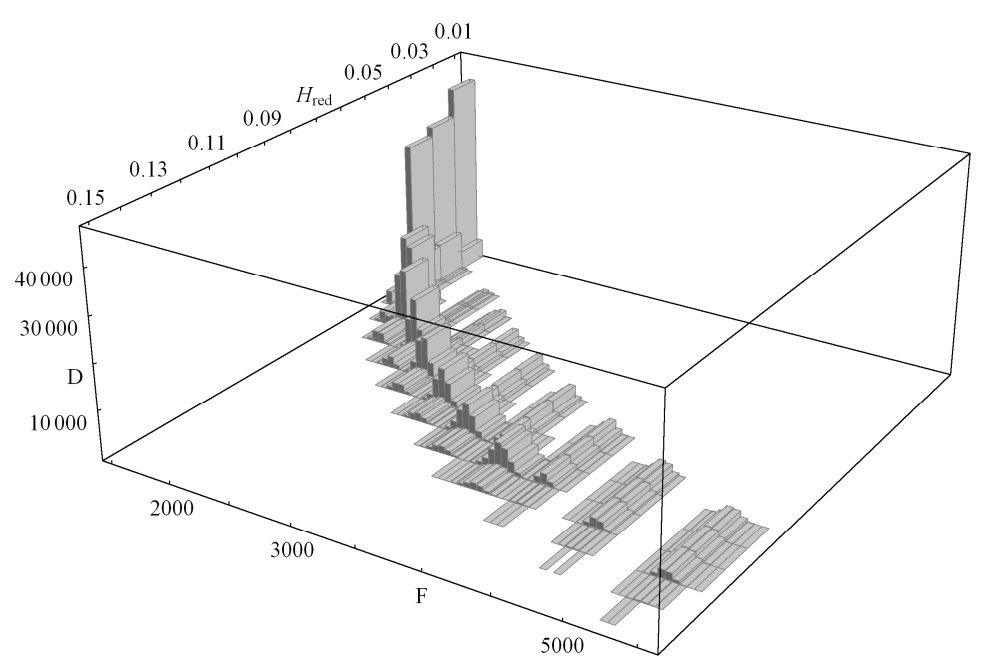

Fig. 4. Empirical distributions of slip loads $F$ for subsequent height reductions $H_{\text {red }}$. Coefficients $\eta$ drawn from the interval [0.1,0.2], the Weibull index $\rho=5$. The figure is based on $5 \times 10^{4}$ samples of $N=50 \times 50$ pillars

Figure 4 illustrates distributions of slip loads $F$ for consecutive system height reductions. The analysed system consists of $N=50 \times 50$ pillars whose strength-thresholds are characterised by the Weibull index $\rho=5$. For initial system height 
reductions $\left(H_{\text {red }} \leq 0.03\right)$ the distribution of slip loads is unimodal. For the next height reductions $\left(H_{r e d}>0.03\right)$ the distribution becomes multimodal. With the increase of $H_{\text {red }}$ the observed interval of slip loads increases and widens.

Chosen distributions of the final loads $F_{f i n}$ have been presented in Figures 5 and 6. It should be noted that the distribution of the final loads $F_{\text {fin }}$ is multimodal. For systems with strengthening coefficients drawn from the interval [0.1, 0.2] values of the final load aggregate around five modes (Fig. 5). In general, the bigger the values of strengthening, the less modes in the distribution (Fig. 6). It has been observed that for a fixed strengthening interval $\left[\eta_{\min }, \eta_{\max }\right]$ the distributions of the final load are characterised by the same number of modes, irrespective of strength-threshold disorder $(\rho=2,5,7,10)$.

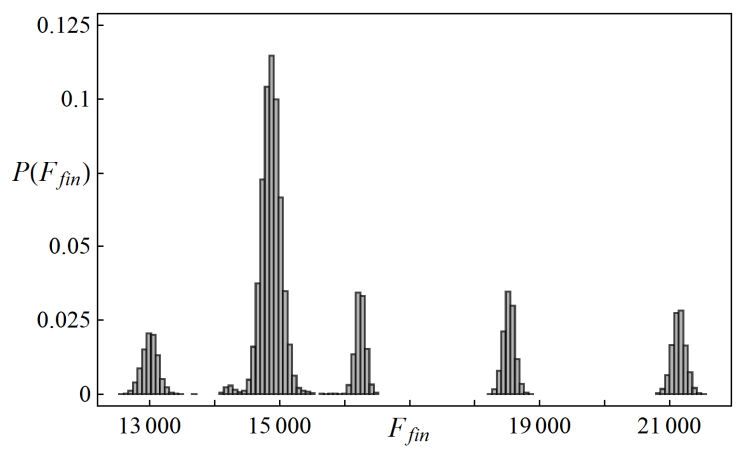

Fig. 5. Empirical probability distribution of the final load $F_{\text {fin }}$ for system of $N=100 \times 100$ pillars. Coefficients $\eta$ drawn from the interval [0.1, 0.2], the Weibull index $\rho=5$. The figure is based on 23750 samples

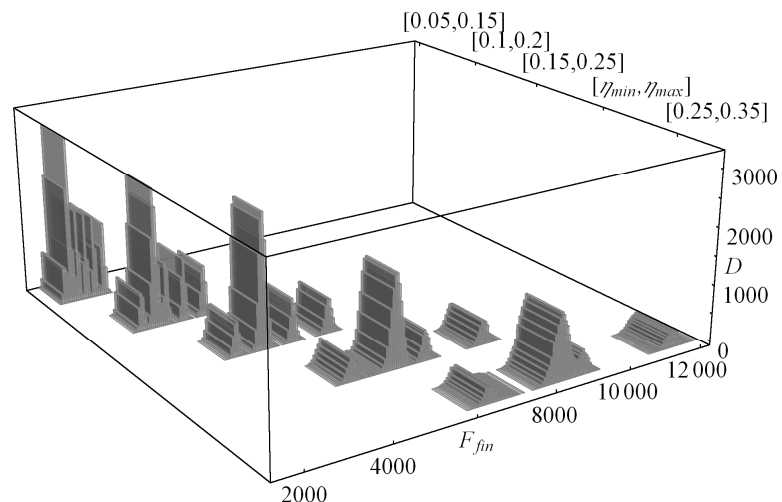

Fig. 6. Empirical distributions of the final loads $F_{\text {fin }}$ for systems of $N=50 \times 50$ pillars and different intervals $\left[\eta_{\min }, \eta_{\max }\right]$. The Weibull index $\rho=2$. Distributions are built on results of $2 \times 10^{4}$ samples for each interval

Here, we define the term of slip avalanche which is the number of pillar height reductions between two consecutive external load increments. $\Delta$ is the size of the 
slip avalanche. Hence the size of the slip avalanche $\Delta$ is the number of pillar height reductions between two stable states. The distribution $D$ of the slip avalanches of sizes $\Delta$ follows a power law distribution $D(\Delta) \sim \Delta^{-\alpha}$. Based on the simulation results a range of variation of exponent $2.3 \leq \alpha \leq 2.35$ has been estimated. Figure 7 presents selected distribution of the slip avalanche sizes. Very long slip avalanches (inclusive number of reductions equal to at least half of the system size) are not included in the above-mentioned figure. The distribution of such long slip avalanches illustrates Figure 8.

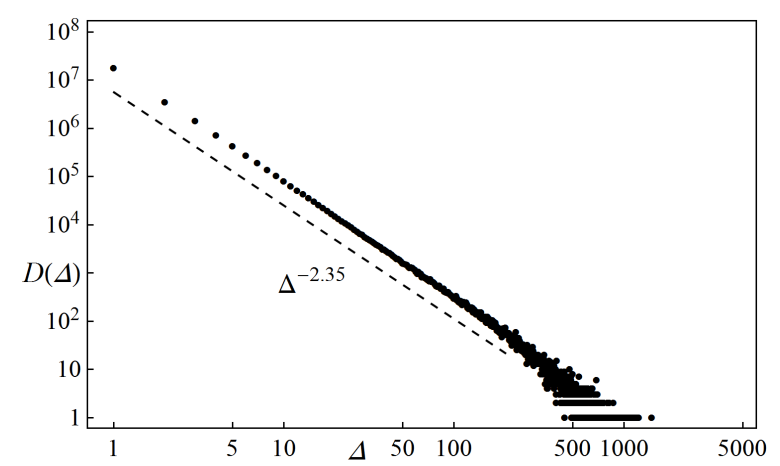

Fig. 7. The distribution of the slip avalanche sizes for systems of $N=150 \times 150$ pillars.

Coefficients $\eta$ drawn from the interval $[0.15,0.25]$, the Weibull index $\rho=5$.

The figure is based on 2415 samples

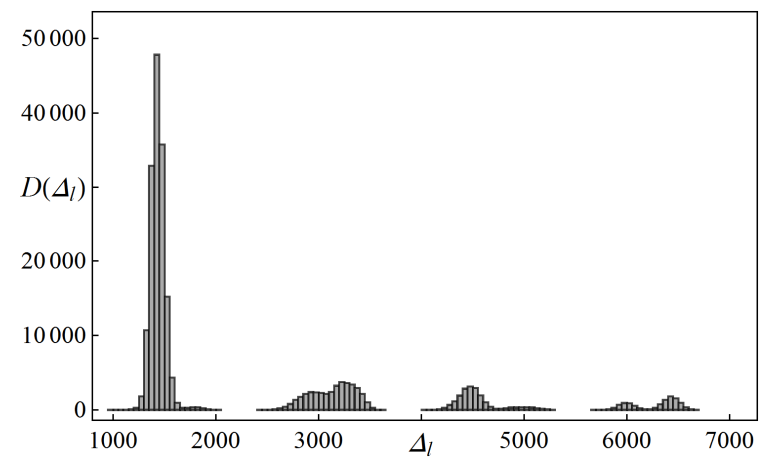

Fig. 8. Empirical distribution of the long slip avalanche sizes for systems of $N=50 \times 50$ pillars. Coefficients $\eta$ drawn from the interval $[0.15,0.25]$, the Weibull index $\rho=5$. The figure is based on $3 \times 10^{4}$ samples

Figure 9 presents probability distribution of a final slip avalanche size $\Delta_{f}$ for the exemplary parameters. As in the case of the final loads $F_{\text {fin }}$ the distribution of $\Delta_{f}$ is multimodal. Comparison of $\Delta_{f}$ distributions for systems with different intervals $\left[\eta_{\min }, \eta_{\max }\right]$ is shown in Figure 10 . From this figure we can say that the bigger the strengthening the smaller the number of modes around which $\Delta_{f}$ are aggregated. 


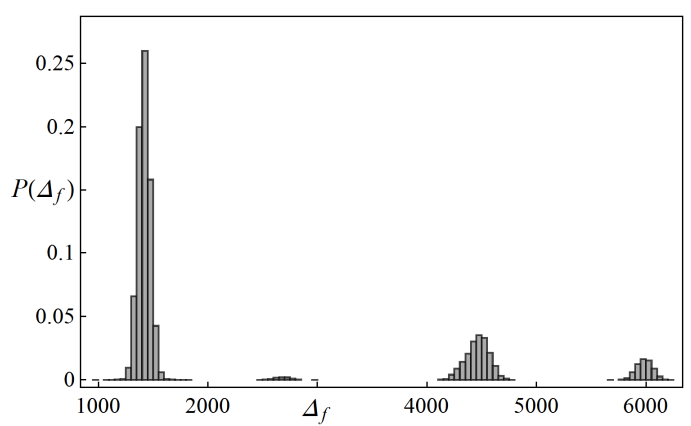

Fig. 9. Empirical probability distribution of the final slip avalanche sizes $\Delta_{f}$ for system of $N=50 \times 50$ pillars. Coefficients $\eta$ drawn from the interval $[0.15,0.25]$,

the Weibull index $\rho=5$. The figure is based on $3 \times 10^{4}$ samples

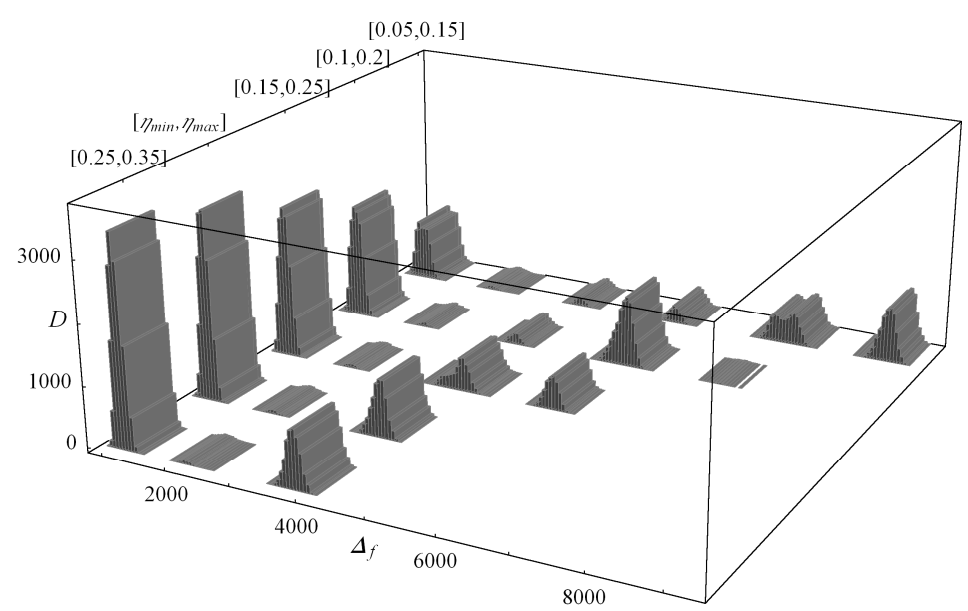

Fig. 10. Empirical distributions of the final slip avalanche sizes $\Delta_{f}$ for systems of $N=50 \times 50$ pillars and different intervals $\left[\eta_{\min }, \eta_{\max }\right]$. The Weibull index $\rho=2$.

Distributions are built on results of $2 \times 10^{4}$ samples for each interval

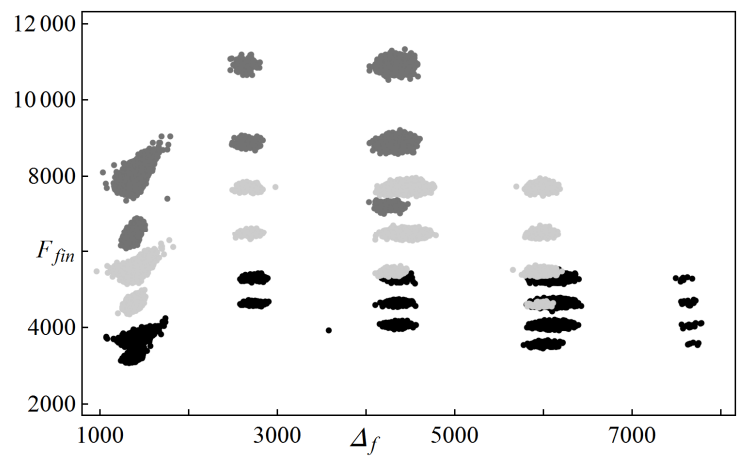

Fig. 11. The final load $F_{f i n}$ vs. size of the final slip avalanche $\Delta_{f}$ for systems of $N=50 \times 50$ pillars and different intervals $\left[\eta_{\min }, \eta_{\max }\right]:[0.1,0.2]$ (black colour), $[0.15,0.25]$ (light grey colour), $[0.2,0.3]$ (dark grey colour). The Weibull index $\rho=5$. The results are based on $3 \times 10^{4}$ samples for each interval 
The dependence between the size of the final slip avalanche $\Delta_{f}$ and the final load $F_{f i n}$ has been shown in Figure 11. As it can be seen, the increases of strengthening cause the growth of the final load and the elimination of the longest final slip avalanches.

\section{Conclusion}

In this paper, we have analysed the partial damage process in quasi-statically loaded arrays of nanopillars. Damage of the system is characterised by a gradual decrease of the system height with many destruction stages of individual pillars. In the presented "mechanical annealing" model the loading of the pillar causes its height reduction and dislocation density drop. Consequently, the pillar strength increases.

Our simulations have been performed for different degrees of the system strength-threshold disorder. We have noticed that the smaller the initial strength-threshold disorder, the greater the mean value of slip loads inducing system height reduction. With a decreasing height of the system, the distributions of slip loads change from unimodal to multimodal.

Damage evolution can also be described by avalanches of pillar height reductions. The distribution of slip avalanche sizes exhibits a power law decay. This power behaviour is not applicable for the longest slip avalanches.

\section{References}

[1] Uchic M.D., Dimiduk D.M., Florando J.N., Nix W.D., Sample dimensions influence strength and crystal plasticity, Science 2004, 305, 986-989.

[2] Jang D., Greer J.R., Transition from a strong-yet-brittle to a stronger-and-ductile state by size reduction of metallic glasses, Nature Materials 2010, 9, 215-219.

[3] Greer J.R., Jang D., Kim J.-Y., Burek M.J., Emergence of new mechanical functionality in materials via size reduction, Adv. Functional Materials 2009, 19, 2880-2886.

[4] Shan Z.W., Mishra R., Syed Asif S.A., Warren O.L., Minor A.M., Mechanical annealing and source-limited deformation in submicrometre-diameter Ni crystals, Nature Materials 2008, 7 , 115-119.

[5] Huang L., Li Q.-J., Shan Z.-W., Li J., Sun J., Ma E., A new regime for mechanical annealing and strong sample-size strengthening in body centered cubic molybdenum, Nature Communications 2011, 2.

[6] Chekurov N., Fabrication process development for silicon micro and nanosystems, $\mathrm{PhD}$ thesis, Aalto University, 2011.

[7] Halasz Z., Kun F., Fiber bundle model with stick-slip dynamics, Phys. Rev. E 2009, 80, 027102.

[8] Halasz Z., Kun F., Slip avalanches in a fiber bundle model, Europhysics Letters 2010, 89, 26008.

[9] Vazquez-Prada M., Gomez J.B., Moreno Y., Pacheco A.F., Time to failure of hierarchical load-transfer models of fracture, Phys. Rev. E 1999, 60, 2581-2594.

[10] Gomez J.B., Vazquez-Prada M., Moreno Y., Pacheco A.F., Bounds for the time to failure of hierarchical systems of fracture, Phys. Rev. E 1999, 59, R1287-R1290. 